\title{
Comportamentos relacionados à saúde entre profissionais de ambulatórios do Sistema Único de Saúde no município de Pelotas-RS*
}

doi: $10.5123 / 51679-49742014000400005$

\author{
Healthcare professionals health-related behaviors in National Health System clinics in the \\ city of Pelotas-RS, Brazil
}

\author{
Alessandra Doumid Borges Pretto \\ Programa de Pós-Graduação em Nutrição e Alimentos, Universidade Federal de Pelotas, Pelotas-RS, Brasil \\ Carla Alberici Pastore \\ Faculdade de Nutrição, Universidade Federal de Pelotas, Pelotas-RS, Brasil
}

Maria Cecília Formoso Assunção

Programa de Pós-Graduação em Nutrição e Alimentos, Universidade Federal de Pelotas, Pelotas-RS, Brasil

\section{Resumo}

Objetivo: descrever comportamentos relacionados à saúde de profissionais de saúde dos ambulatórios do Sistema Único de Saúde (SUS) no município de Pelotas-RS, Brasil, em relação a alimentação, consumo de bebidas alcoólicas, tabagismo e prática de atividade física. Métodos: estudo transversal com profissionais que trabalhavam no atendimento direto a pacientes, de maio a julho de 2012. Resultados: dos 340 profissionais, 53,0\% eram mulheres com média de idade de 42 anos (desviopadrão: 13,7 anos), em sua maioria (68,9\%) eutróficas, enquanto 50,6\% dos homens abordados apresentavam sobrepeso; 49,7\% eram sedentários e 27,6\% consumiam frutas diariamente; a presença de um 'perfil saudável' (concomitância de índice de massa corporal normal, não fumar, consumir frutas e verduras diariamente, praticar atividade física e não adicionar sal aos alimentos já preparados) esteve presente em 15,3\% dos profissionais e foi mais frequente entre as mulheres $(\mathrm{p}=0,004)$. Conclusão: são necessárias mudanças nos comportamentos relacionados à saúde dos profissionais estudados.

Palavras-chave: Alimentação; Pessoal de Saúde; Doença Crônica; Estudos Transversais.

\begin{abstract}
Objective: to describe health-related behavior of health professionals at Unified Health System (SUS) outpatient clinics in the city of Pelotas - RS. Brazil, in relation to eating habits, alcohol consumption, smoking and physical activity. Methods: this was a cross-sectional study with professionals working in direct patient care, from May to July 2012. Results: of the 340 professionals, 53.0\% were women, with a mean age of 42 years (standard deviation 13.7 years). Most women (68.9\%) were eutrophic, while $50.6 \%$ of men were overweight; $49.7 \%$ were sedentary and $27.6 \%$ ate fruit daily. A "bealthy profile" (concomitant normal BMI, not smoking, eating fruit and vegetables daily, regular physical activity and not adding additional salt to food), was found in $15.3 \%$ of professionals and was more frequent among women $(p=0,004)$. Conclusion: the majority of the professionals studied need to change their behaviors.
\end{abstract}

Key words: Feeding; Health Personnel; Chronic Disease; Cross-Sectional Studies.

\footnotetext{
* Artigo elaborado a partir da dissertação de Mestrado da autora principal, Alessandra Doumid Borges Pretto, defendida junto ao Programa de Pós-Graduação em Nutrição e Alimentos da Universidade Federal de Pelotas, Pelotas-RS, em fevereiro de 2013. À autora principal, foi concedida bolsa de Mestrado pela Coordenação de Aperfeiçoamento de Pessoal de Nível Superior Fundação Capes/Ministério da Educação.
}

Endereço para correspondência:

Alessandra Doumid Borges Pretto - Rua General Teles, n 645, apto. 302, Centro, Pelotas-RS, Brasil. CEP: $96010-310$

E-mail:alidoumid@yahoo.com.br 


\section{Introdução}

Nos últimos anos, tem-se observado um aumento acelerado da prevalência de sobrepeso e de obesidade na população geral, tanto nos países desenvolvidos como em desenvolvimento. A Organização Mundial da Saúde (OMS) afirma que a obesidade está diretamente relacionada ao surgimento das doenças crônicas não transmissíveis (DCNT) e que estas representam a principal causa de mortalidade, sendo responsáveis por $63 \%$ de todas as mortes no mundo. ${ }^{1}$ Entre as principais causas de DCNT, está a alimentação inadequada.

De acordo com a OMS, um pequeno conjunto de fatores de risco modificáveis responde pela grande maioria das mortes e por fração importante da carga de DCNT. Entre esses fatores, encontram-se o tabagismo, o sedentarismo e as dislipidemias, associadas principalmente ao consumo excessivo de gorduras de origem animal. ${ }^{1}$

\section{É possível que as atitudes dos profissionais da saúde, com o próprio exemplo de seus bons hábitos de vida e de saúde, tenham impacto na saúde coletiva.}

Muitos países, entre eles o Brasil, definiram a promoção da alimentação saudável como uma das principais estratégias para o controle dessas doenças. ${ }^{1}$ Nesse sentido, em 2006, o Ministério da Saúde elaborou os '10 Passos para a Alimentação Saudável', ${ }^{2}$ ação que consta da Política Nacional de Alimentação e Nutrição (PNAN), cujos objetivos são aumentar o nível de conhecimento da população sobre a importância da promoção da saúde, da manutenção de peso saudável e de ter uma vida ativa, além de modificar atitudes e práticas sobre alimentação e atividade física e prevenir o excesso de peso. Os sete primeiros passos do PNAM estão relacionados à dieta, e os três últimos, ao incentivo para uma vida mais ativa. ${ }^{2}$

Um dos principais aspectos implicados na melhoria da qualidade de vida de uma população é o aumento de sua capacidade de compreender os fenômenos relacionados a seu bem-estar, sendo os profissionais de saúde multiplicadores dessas informações na sociedade. ${ }^{3}$ É possível que as atitudes desses profissionais, com o próprio exemplo de seus bons hábitos de vida e de saúde, tenham impacto na saúde coletiva além das orientações oferecidas a seus pacientes. ${ }^{4}$

0 presente estudo teve por objetivo descrever os comportamentos relacionados à saúde dos profissionais que prestam atendimento nos ambulatórios do Sistema Único de Saúde (SUS) do município de Pelotas, estado do Rio Grande do Sul, em relação a alimentação, consumo de bebidas alcoólicas, tabagismo e prática regular de atividade física.

\section{Métodos}

Estudo transversal realizado nos ambulatórios (serviços de atenção secundária) que atendiam pelo Sistema Único de Saúde (SUS) no município de Pelotas, região sul do estado do Rio Grande do Sul.

Em 2014, Pelotas-RS possuía uma população de 328.275 habitantes. A estrutura de Saúde Pública municipal era constituída por uma rede de atenção primária com 51 unidades básicas de saúde, uma rede de atenção secundária representada por seis ambulatórios especializados, seis centros de atenção psicossocial e um pronto-socorro, e um nível terciário de atenção formado por cinco hospitais gerais, sendo dois universitários e três filantrópicos. ${ }^{5}$

A população em estudo foi composta por todos os profissionais que atuavam diretamente no atendimento aos pacientes da rede de atenção secundária à saúde, no período de maio a julho de 2012, selecionado por conveniência. Todos os profissionais - médicos, enfermeiros, nutricionistas, fisioterapeutas e psicólogos - foram convidados a participar do estudo. Os ambulatórios não contavam com a presença de odontólogos.

Foram consideradas recusas ou perdas os profissionais que não quiseram participar da pesquisa ou não foram encontrados pelos entrevistadores após três tentativas de contato, em dias e horários distintos, para aplicação do questionário, criado especificamente para o estudo.

Como passo inicial, os profissionais foram identificados e convidados a participar da pesquisa. Após sua concordância quanto à participação e assinatura do Termo de Consentimento Livre e Esclarecido, eles responderam a um questionário estruturado contendo perguntas sobre alimentação, consumo de bebidas alcoólicas, presença de DCNT, frequência de atividade física e hábito de fumar. 0 questionário foi aplicado por alunos da Faculdade de Nutrição da Universidade Federal de Pelotas (UFPEL), previamente treinados 
para essa função e para a aferição das medidas antropométricas, ambas supervisionadas, durante o período de coleta dessas informações, pela pesquisadora principal (Pretto ADB). 0 protocolo de pesquisa foi previamente testado em estudo-piloto, realizado em um ambulatório privado do município.

Foram coletadas informações sociodemográficas (sexo; e faixa etária, nas categorias de 20 a 39, 40 a 59 e 60 ou mais anos) e aferidos peso e altura. Para a pesagem, foi utilizada balança digital da marca Tanita ${ }^{\circledR}$ modelo PL 150, com capacidade até $150 \mathrm{~kg}$ e precisão de 100g. Para aferição da altura, foi utilizado um estadiômetro portátil de alumínio, com precisão de um milímetro. A aferição do peso e da altura foi realizada com o indivíduo trajando roupas leves e sem sapatos, de acordo com técnica padronizada pela OMS. ${ }^{6}$

0 índice de massa corporal (IMC) foi calculado mediante a divisão do peso pela altura em metros ao quadrado, sendo os indivíduos classificados da seguinte forma: IMC menor de $18,5 \mathrm{~kg} / \mathrm{m}^{2}$, baixo peso; de 18,5 a $24,9 \mathrm{~kg} / \mathrm{m}^{2}$, eutrofia; maior de 24,9 até $29,9 \mathrm{~kg} / \mathrm{m}^{2}$, sobrepeso; e IMC acima de $29,9 \mathrm{~kg} /$ $\mathrm{m}^{2}$, obesidade. ${ }^{6}$

Considerou-se como tabagismo ter fumado no mínimo um cigarro no último mês. 0 consumo de bebidas alcoólicas foi categorizado em diário, de 1 a 3 vezes na semana, de 4 a 6 vezes na semana ou esporadicamente. ${ }^{7}$ A prática regular de atividade física foi considerada como o relato da prática de exercícios físicos, coletando-se informação sobre sua frequência de realização: prática diária; esporádica; de 1 a 3 vezes na semana; ou de 4 a 6 vezes na semana. ${ }^{7}$ Tanto 0 consumo de álcool quanto a prática de atividade física foram categorizados de maneira a permitir respostas rápidas, haja vista o pouco tempo disponível para a aplicação do questionário. Neste, perguntou-se aos profissionais sobre a presença de doenças crônicas não transmissíveis, como diabetes, hipertensão arterial sistêmica, doenças cardiovasculares, câncer, doenças renais e doenças respiratórias, musculoesqueléticas e neuropsiquiátricas, além de outras referidas pelos próprios entrevistados, como hipotireoidismo, artrite reumatoide, esclerodermia, síndrome de ovários policísticos e tireoidite de Hashimoto.

A informação sobre hábitos alimentares foi obtida por meio de um questionário, no qual, primeiramente, perguntava-se sobre o consumo de determinado alimento (sim/não); para as respostas afirmativas a cada uma dessas perguntas, era questionada a frequência de consumo semanal.

Foram consideradas as seguintes recomendações de hábitos alimentares saudáveis: consumo de três porções de frutas e vegetais ao dia; consumo de três porções de leite e derivados ao dia; consumo de uma porção de carne e/ou ovo ao dia; consumo de açúcares e doces; acréscimo de sal à refeição pronta; consumo de alimentos do grupo de óleos e gorduras; e consumo de dois litros de água por dia. ${ }^{7}$ Como parâmetro para as variáveis de hábitos alimentares e de vida, utilizou-se o Guia Alimentar para a População Brasileira, elaborado pelo Ministério da Saúde. ${ }^{?}$

Foi construída uma variável denominada 'perfil saudável', para a qual foi considerada a concomitância entre as seguintes características: IMC dentro da normalidade (18,5 a 24,9kg/m²); não fumar; consumir frutas e verduras diariamente; praticar atividade física regularmente; e não adicionar sal às refeições ou alimentos já preparados. ${ }^{7}$

0 controle de qualidade da coleta de dados foi realizado com 34 indivíduos ( $10 \%$ da amostra), selecionados aleatoriamente. Nessa oportunidade, foi aplicado pela pesquisadora principal um questionário reduzido e estruturado, para testar a repetibilidade das respostas às seguintes variáveis: fumo; ingestão de bebida alcoólica; prática regular de atividade física; e ingestão diária de carnes e/ou ovos. 0 resultado do teste Kappa para todas as questões foi de 1.0, considerado como de concordância perfeita.

Os questionários foram digitados em banco construído no programa Epi Info $6.05 \mathrm{~d}^{\circledR}$, após serem codificados e revisados. Os dados foram processados com dupla digitação e em seguida, efetuou-se a checagem de consistência das informações.

As análises estatísticas foram realizadas por meio do pacote estatístico Stata $12.0^{\circledR}$, admitindo-se um nível de significância de 5\%, bicaudal. Primeiramente, foi realizada a descrição dos dados e logo, empregado o teste qui-quadrado de Pearson ou teste exato de Fischer - segundo o mais adequado -, para verificar a associação entre exposições e desfechos.

0 projeto do estudo foi aprovado pelo Comitê de Ética em Pesquisa da Universidade Federal de Pelotas (OF. 28/12, de 25 de abril de 2012). A pesquisa foi realizada de acordo com a Resolução do Conselho Nacional de Saúde (CNS) n ${ }^{\circ}$ 466, de 12 de dezembro de 2012. 


\section{Resultados}

Dos 348 profissionais que trabalhavam nos seis ambulatórios que atendiam pelo SUS no município de Pelotas-RS, 340 (97,7\%) participaram da pesquisa. Dos profissionais que não participaram, 2 recusaram e 6 não puderam ser entrevistados, pois estavam de férias e/ou de licença do trabalho no período da coleta dos dados.

Entre os profissionais avaliados, 53,0\%(180) eram mulheres. A idade média foi de 42 anos (desvio-padrão: $\mathrm{DP}=13,7$ anos), sendo a idade mínima de 22 anos e a máxima de 80 anos. A maioria dos profissionais eram médicos $(85,3 \%)$, seguidos de nutricionistas
$(5,6 \%)$, enfermeiros (3,5\%) e fisioterapeutas (3,2\%). A categoria profissional menos frequente foi a de psicólogo $(2,4 \%)$ (Tabela 1).

Apenas $0,8 \%$ dos profissionais estudados referiu ingerir bebida alcoólica diariamente e 5,0\% fumar. Metade dos profissionais referiu praticar atividade física regularmente: 50,3\% (Tabela 1).

Aproximadamente um terço dos profissionais referiu apresentar DCNT, sendo a hipertensão arterial sistêmica e doenças respiratórias as mais referidas (Tabela 2).

A Tabela 3 descreve os hábitos alimentares dos profissionais: $63,8 \%$ deles relataram consumo diário

\section{Tabela 1 - Caracterização sociodemográfica e dos comportamentos relacionados à saúde entre os profissionais de ambulatórios ( $n=340)$ do Sistema Único de Saúde (SUS) em Pelotas, Rio Grande do Sul, 2012}

\begin{tabular}{|c|c|c|}
\hline Variáveis & $\mathbf{n}$ & $\%$ \\
\hline \multicolumn{3}{|l|}{ Sexo } \\
\hline Feminino & 180 & 53,0 \\
\hline Masculino & 160 & 47,0 \\
\hline \multicolumn{3}{|l|}{ Idade } \\
\hline 20 a 39 anos & 181 & 53,2 \\
\hline 40 a 59 anos & 118 & 34,7 \\
\hline 60 ou mais & 41 & 12,1 \\
\hline \multicolumn{3}{|l|}{ Formação profissional } \\
\hline Médico & 290 & 85,3 \\
\hline Nutricionista & 19 & 5,6 \\
\hline Enfermeiro & 12 & 3.5 \\
\hline Fisioterapeuta & 11 & 3,2 \\
\hline Psicólogo & 8 & 2,4 \\
\hline \multicolumn{3}{|l|}{ Tabagismo } \\
\hline Não & 323 & 95,0 \\
\hline Sim & 17 & 5,0 \\
\hline \multicolumn{3}{|l|}{ Consumo de bebida alcoólica } \\
\hline Não & 91 & 26,8 \\
\hline Sim & 249 & 73,2 \\
\hline \multicolumn{3}{|c|}{ Frequência do consumo de bebida alcoólica } \\
\hline Diariamente & 2 & 0,8 \\
\hline 1 a 3 vezes/semana & 114 & 45,8 \\
\hline 4 a 6 vezes/semana & 7 & 2,8 \\
\hline Raramente ou eventualmente & 126 & 50,6 \\
\hline \multicolumn{3}{|l|}{ Prática de atividade física regular } \\
\hline Não & 169 & 49,7 \\
\hline Sim & 171 & 50,3 \\
\hline \multicolumn{3}{|c|}{ Frequência da prática de atividade física } \\
\hline Diariamente & 14 & 8,2 \\
\hline 1 a 3 vezes na semana & 118 & 69,0 \\
\hline 4 a 6 vezes na semana & 36 & 21,1 \\
\hline Raramente ou eventualmente & 3 & 1,7 \\
\hline Presença do "perfil saudável"a & 52 & 15,3 \\
\hline
\end{tabular}


Tabela 2 - Prevalência de doenças crônicas não transmissíveis autorreferidas pelos profissionais de ambulatórios ( $\mathrm{n=340)}$ do Sistema Único de Saúde em Pelotas, Rio Grande do Sul, 2012

\begin{tabular}{lcc}
\hline Doenças crônicas & $\mathrm{N}$ & $\%$ \\
\hline Não & 222 & 65,3 \\
Sim & 118 & 34,7 \\
\hline Principais doenças crônicas não transmissíveis (DCNT) & & 9,3 \\
\hline Diabetes mellitus & 11 & 39,0 \\
Hipertensão & 46 & 3,4 \\
Câncer & 4 & 25,4 \\
Doença respiratória & 30 & 6,8 \\
Doença cardiovascular & 8 & 1,7 \\
Doença renal & 2 & 3,4 \\
Doença musculoesquelética & 4 & 0,8 \\
Doença neuropsiquiátrica & 1 & 10,2 \\
Outras doenças ${ }^{\text {a }}$ & 12 & 100,0 \\
\hline TOTAL & 340 & \\
\hline
\end{tabular}

a) Outras doenças incluem: hipotireoidismo, artrite reumatoide, esclerodermia, síndrome de ovários policísticos e tireoidite de Hashimoto.

de frutas e $82,3 \%$ consumiam verduras e/ou legumes diariamente. 0 consumo diário de leite e derivados foi referido por $79,1 \%$ dos entrevistados.

Dos profissionais estudados, $48,0 \%$ afirmaram que raramente consumiam frituras ou gordura aparente de carnes, $28,2 \%$ consumiam doces diariamente, $16,8 \%$ referiram fracionar sua alimentação diária em 5 vezes ou mais e 50,9\% ingeriam menos de 4 copos de água por dia (Tabela 3).

A maioria dos profissionais eram eutróficos (51,8\%), 35,9\% apresentaram sobrepeso e $11,8 \%$ obesidade. 0 excesso de peso foi maior entre os homens $(67,5 \%)$, em relação às mulheres $(30,0 \%)$ $(\mathrm{p}<0,001)$ (Tabela 4).

A prevalência de 'perfil saudável' foi de 15,3\% entre os profissionais e esteve significativamente associada apenas ao sexo, sendo observada com maior frequência entre as mulheres $(20,5 \%)$, na comparação com os homens $(9,3 \%)(\mathrm{p}=0,004)$.

\section{Discussão}

Entre os profissionais atuantes nos ambulatórios do SUS do município de Pelotas-RS, no período de maio a julho de 2012, observou-se elevada prevalência de sedentarismo e de sobrepeso ou obesidade, fatores que podem atuar de forma negativa sobre a saúde. ${ }^{8}$ Deve-se considerar que cerca de um terço desses profissionais referiu-se a si mesmo como portador de alguma DCNT.
Menos de um quinto dos profissionais reunia algumas características consideradas importantes para a manutenção de uma boa saúde, conjuntamente denominadas aqui de 'perfil saudável'.

Quase a metade dos profissionais entrevistados apresentou excesso de peso ou obesidade. De forma similar, estudo realizado com plantonistas de unidades de saúde de Teresina-PI mostrou que 53,7\% deles apresentavam sobrepeso. ${ }^{8}$ No presente estudo, as prevalências de sobrepeso e obesidade foram superiores no sexo masculino, de maneira semelhante a outros estudos..$^{9,10}$

A inatividade física e o baixo nível de condicionamento físico têm sido considerados fatores de risco para mortalidade prematura, nesse sentido tão importantes quanto fumar ou apresentar dislipidemia e hipertensão arterial. ${ }^{11}$ Hallal e colaboradores mostraram que a inatividade física aumenta o risco de muitas condições adversas à saúde, incluindo as principais DCNT, como doenças coronarianas, diabetes tipo 2, câncer de mama e de cólon, além de reduzir a expectativa de vida. ${ }^{12}$ É digno de nota, portanto, o elevado número de profissionais sedentários observado neste e em outros estudos com trabalhadores de saúde. ${ }^{8,9,13}$

0 presente estudo também evidenciou baixa frequência de tabagismo entre os profissionais entrevistados. Teixeira e cols. encontraram frequência de tabagismo de $10 \%$ em médicos da cidade de 
Tabela 3 - Caracterização quanto aos hábitos alimentares, fracionamento das refeições e consumo de água pelos profissionais de ambulatórios ( $n=340$ ) do Sistema Único de Saúde (SUS) em Pelotas, Rio Grande do Sul, 2012

\begin{tabular}{|c|c|c|}
\hline Hábitos alimentares & $\mathbf{n}$ & $\%$ \\
\hline \multicolumn{3}{|l|}{ Consumo diário de frutas e/ou suco de frutas } \\
\hline Não & 123 & 36,2 \\
\hline Sim & 217 & 63,8 \\
\hline \multicolumn{3}{|l|}{ Consumo de frutas e/ou suco de frutas por dia } \\
\hline 1 unidade e/ou 1 copo ou 1 porção & 76 & 35,1 \\
\hline 2 unidades e/ou 2 copos ou 2 porções & 81 & 37,3 \\
\hline 3 unidades e/ou 3 copos ou 3 porções & 60 & 27,6 \\
\hline \multicolumn{3}{|l|}{ Consumo diário de legumes e verduras } \\
\hline Não & 60 & 17,7 \\
\hline Sim & 280 & 82,3 \\
\hline \multicolumn{3}{|l|}{ Consumo de legumes e verduras por dia } \\
\hline Menos de 3 colheres de sopa ou 1 porção & 118 & 42,2 \\
\hline De 4 a 7 colheres de sopa ou 2 porções & 127 & 45,3 \\
\hline 8 ou mais de 8 colheres de sopa ou 3 porções & 35 & 12,5 \\
\hline \multicolumn{3}{|l|}{ Consumo de produtos integrais } \\
\hline Diariamente & 149 & 43,8 \\
\hline 1 a 3 vezes na semana & 88 & 25,9 \\
\hline 4 a 6 vezes na semana & 24 & 7,1 \\
\hline Raramente ou eventualmente & 79 & 23,2 \\
\hline \multicolumn{3}{|l|}{ Consumo diário de carne e/ou ovo } \\
\hline Não & 39 & 11,5 \\
\hline Sim & 301 & 88,5 \\
\hline \multicolumn{3}{|l|}{ Consumo de carne e/ou ovo por dia } \\
\hline 1 pedaço/fatia/colher de sopa ou 1 ovo & 138 & 45,9 \\
\hline 2 pedaços/fatias/colheres de sopa ou 2 ovos & 125 & 41,5 \\
\hline 3 ou mais pedaços/fatias/colheres de sopa ou 3 ovos & 38 & 12,6 \\
\hline \multicolumn{3}{|l|}{ Consumo diário de leite e derivados } \\
\hline Não & 71 & 20,9 \\
\hline Sim & 269 & 79,1 \\
\hline \multicolumn{3}{|l|}{ Consumo de leite e derivados por dia } \\
\hline 1 copo de leite ou iogurte & 100 & 37,2 \\
\hline 2 copos de leite ou iogurte & 119 & 44,2 \\
\hline 3 copos ou mais de leite ou iogurte & 50 & 18,6 \\
\hline \multicolumn{3}{|l|}{ Consumo de frituras e gordura aparente das carnes } \\
\hline Raramente ou nunca & 163 & 48,0 \\
\hline Todos os dias & 12 & 3,5 \\
\hline De 1 a 3 vezes na semana & 149 & 43,8 \\
\hline De 4 a 6 vezes na semana & 16 & 4,7 \\
\hline \multicolumn{3}{|l|}{ Consumo de temperos industrializados e embutidos } \\
\hline Raramente ou nunca & 106 & 31,1 \\
\hline Todos os dias & 58 & 17,1 \\
\hline De 1 a 3 vezes na semana & 139 & 40,9 \\
\hline De 4 a 6 vezes na semana & 37 & 10,9 \\
\hline \multicolumn{3}{|l|}{ Consumo de doces, refrigerantes e sucos industrializados } \\
\hline Raramente ou nunca & 74 & 21,8 \\
\hline Todos os dias & 96 & 28,2 \\
\hline De 1 a 3 vezes na semana & 116 & 34,1 \\
\hline De 4 a 6 vezes na semana & 54 & 15,9 \\
\hline \multicolumn{3}{|l|}{ Acréscimo de sal na refeição pronta } \\
\hline Não & 275 & 80,9 \\
\hline Sim & 65 & 19,1 \\
\hline \multicolumn{3}{|l|}{ Número de refeicões por dia } \\
\hline 2 & 36 & 10,6 \\
\hline 3 a 4 & 247 & 72,6 \\
\hline 5 ou mais & 57 & 16,8 \\
\hline \multicolumn{3}{|l|}{ Consumo diário de água (em copos) } \\
\hline Menos de 4 & 173 & 50,9 \\
\hline 4 a 8 & 122 & 35,9 \\
\hline Mais de 8 & 45 & 13,2 \\
\hline
\end{tabular}




\section{Tabela 4 - Estado nutricional classificado pelo índice de massa corporal (IMC) segundo sexo dos profissionais de} ambulatórios ( $n=340$ ) do Sistema Único de Saúde (SUS) em Pelotas, Rio Grande do Sul, 2012

\begin{tabular}{|c|c|c|c|c|c|}
\hline \multirow{2}{*}{$\begin{array}{l}\mathrm{IMC}\left(\mathrm{Kg} / \mathrm{m}^{2}\right) \\
\text { Categoria }\end{array}$} & \multirow{2}{*}{\multicolumn{2}{|c|}{ Homens }} & \multicolumn{2}{|c|}{ Mulheres } & \multirow{2}{*}{ Valor $\mathrm{p}^{\mathrm{a}}$} \\
\hline & & & & $\%$ & \\
\hline Baixo peso ${ }^{b}$ & - & - & 2 & 1,1 & \multirow{4}{*}{$<0,001$} \\
\hline Eutrofiac & 52 & 32,5 & 124 & 68,9 & \\
\hline Sobrepeso $^{d}$ & 81 & 50,6 & 41 & 22,8 & \\
\hline Obesidade $^{e}$ & 27 & 16,9 & 13 & 7,2 & \\
\hline
\end{tabular}

a) Teste exato de Fischer

b) Baixo peso: IMC: $<18,5 \mathrm{~kg} / \mathrm{m}^{2}$

c) Eutrofia: 18,5 a $24,9 \mathrm{~kg} / \mathrm{m}^{2}$

d) Sobrepeso: 25 a $29,9 \mathrm{~kg} / \mathrm{m}^{2}$

e) Obesidade: $\geq 30,0 \mathrm{~kg} / \mathrm{m}^{2}$

Barbacena-MG. ${ }^{14}$ Outra pesquisa, conduzida por Realpe e colaboradores, ao avaliar trabalhadores de saúde na Colômbia, encontrou uma proporção de $12,5 \%$ de fumantes. ${ }^{15}$

Dos profissionais que ingeriam bebidas alcoólicas, apenas 3\% admitiam um consumo regular de 4 a 6 vezes na semana, e menos de $1 \%$ consumo diário. Este achado foi similar ao de Bazargan e cols., segundo quem, $6 \%$ de médicos da Califórnia, Estados Unidos da América, relataram consumo regular de bebidas alcoólicas. ${ }^{16}$ No presente estudo, não foi possível avaliar o tipo e a quantidade de bebida alcoólica ingerida, motivo pelo qual esse item não foi incluído na construção da variável 'perfil saudável'.

Entre os profissionais da Saúde de Pelotas-RS entrevistados, 34,7\% referiram diagnóstico de pelo menos uma doença crônica. Jansen e cols., em estudo com trabalhadores de um hospital universitário de Belo Horizonte-MG, encontraram um percentual bastante inferior, situando-se em torno de $9 \% .{ }^{17}$

Nos últimos 20 anos, no Brasil e no mundo, observa-se uma importante mudança no perfil de mortalidade da população, caracterizado pelo aumento de óbitos causados por DCNT, ${ }^{1}$ com destaque para a hipertensão arterial sistêmica, ${ }^{18}$ cuja prevalência estimada na população brasileira acima de 40 anos de idade é de $35 \% .{ }^{18}$ Neste estudo, a hipertensão foi a DCNT mais referida pelos profissionais, presente em $13,5 \%$ dos indivíduos. Na população geral, a prevalência de hipertensão é maior; porém sabe-se que ela aumenta com a idade, ${ }^{19}$ e como a média de idade neste estudo foi de 42 anos, esse comporta- mento da doença relacionado à idade pode explicar - ainda que parcialmente - a menor prevalência de hipertensão entre os trabalhadores avaliados. Valores semelhantes foram encontrados no estudo desenvolvido por Realpe e cols. junto a trabalhadores de saúde da Colômbia, onde 11,5\% eram hipertensos, ${ }^{15}$ e no estudo de Teixeira e cols. com médicos de Barbacena-MG, que referiu uma prevalência de hipertensão arterial sistêmica de $22 \%{ }^{14}$

Cerca de $40 \%$ dos profissionais relataram consumir produtos integrais e ricos em fibras diariamente. Santos e cols. afirmam que o elevado consumo de fibras associa-se a um menor risco de doença cardiovascular e diabetes mellitus tipo 2, sendo importante também para o controle desse agravo. ${ }^{20}$

De acordo com o Guia Alimentar para a População Brasileira, o consumo de frutas e hortaliças deve ser, no mínimo, de 6 porções ou de 400 a 600 gramas por dia. ${ }^{7}$ Neste estudo, apenas um terço dos profissionais informaram consumir a quantidade de hortaliças e frutas recomendada. Quanto ao consumo de carne e/ ou ovo, apenas metade dos entrevistados consumia a quantidade recomendada (uma porção diária) $;^{7}$ os demais consumiram além da recomendação, o que pode levar a um maior risco de doença cardiovascular e alguns tipos de câncer. ${ }^{4,21}$ Sobre o consumo de leite e derivados, somente $18,6 \%$ seguiam a recomendação de ingerir três porções ao dia, resultado semelhante ao encontrado por Marcondelli e cols. ${ }^{22}$

Apesar de o consumo diário de doces e outros alimentos ricos em açúcar, frituras e gordura aparente das carnes não ter sido preocupante, achado 
semelhante aos de outros estudos, ${ }^{9,22}$ observou-se baixo consumo de alimentos ricos em fibra, leite e derivados e alto consumo de carnes, hábitos que podem levar à ingestão excessiva de sódio, álcool, açúcares, gorduras e alimentos de alta densidade energética, correlacionados a excesso de peso e elevado risco cardiovascular. ${ }^{23}$

A recomendação de fracionar a alimentação em três refeições e dois lanches diários foi seguida por apenas $16,8 \%$ dos entrevistados. Diversos estudos têm indicado a relação entre $o$ fracionamento das refeições e uma alimentação saudável. ${ }^{9,20} \mathrm{Em}$ estudo realizado no Rio de Janeiro, Oliveira \& Sichieri mostraram que o maior fracionamento de refeições constitui importante medida de prevenção e controle da dislipidemia e, consequentemente, da prevenção de doença cardiovascular. ${ }^{24}$

Metade dos entrevistados relatou ingerir menos de 4 copos de água ao dia, configurando baixa ingestão. ${ }^{23}$ Contudo, não foi avaliado o hábito de ingerir chimarrão - ou mate, bebida comum entre os gaúchos -, que precisa ser considerado quando analisado 0 consumo hídrico entre habitantes do Sul do Brasil, particularmente.

Comportamentos relacionados à saúde foram pouco frequentes entre os profissionais avaliados, como a prática regular de atividade física, alto consumo de fibras, leite e derivados. Apesar de os profissionais de saúde possuírem conhecimento sobre hábitos saudáveis de vida, ainda se observa uma distância entre o conhecimento e o comportamento referidos por eles. Estudo realizado por Sebold, Radunz e Carraro mostrou que embora tenham conhecimento sobre hábitos saudáveis, esses profissionais apresentam grande dificuldade em manter e persistir esses hábitos, propiciando o sedentarismo, o aparecimento de doenças e 0 aumento de peso. ${ }^{25}$

Os resultados deste estudo podem ser resumidos na seguinte constatação: quando avaliada a presença do 'perfil saudável' entre os profissionais de saúde, poucos se enquadraram nesse perfil. A maior frequência de hábitos saudáveis no sexo feminino mostra maior conscientização das mulheres sobre a importância de adotar e manter bons hábitos de vida e saúde, resultado que condiz com o apontado pelo Ministério da Saúde ao afirmar que, de uma forma geral, as mulheres são mais cuidadosas com sua saúde, procuram mais pelos serviços de saúde e sofrem menos com o excesso de peso. ${ }^{26}$

Este estudo teve como limitação a possibilidade de ocorrência do viés de resposta. Se os resultados apresentados já são preocupantes, todavia é provável que alguns profissionais tenham-se omitido da autorreferência de hábitos não saudáveis em suas respostas, pelo fato de serem conhecedores dos hábitos de vida adequados. Outro fator limitante deste estudo foi seu caráter transversal, de modo que as informações sobre desfechos e exposições referem-se ao momento da coleta de dados, não sendo possível estabelecer uma relação de temporalidade entre as variáveis.

As condições de saúde observadas apontam para a necessidade de mudanças nos hábitos de vida dos profissionais estudados. Recomenda-se que ações concernentes ao programa de Saúde do Trabalhador sejam formalmente incluídas na agenda da rede de Atenção Básica do Sistema Único de Saúde. O local de trabalho constitui um ambiente adequado para 0 desenvolvimento de intervenções visando à promoção da saúde e qualidade de vida. ${ }^{27}$

\section{Contribuiç̧̃es das autoras}

Pretto ADB e Pastore CA contribuíram na concepção e delineamento do estudo, redação e revisão crítica do conteúdo intelectual.

Assunção MC contribuiu na análise e interpretação dos resultados, redação e revisão crítica do conteúdo.

Todas as autoras aprovaram a versão final do manuscrito e declaram serem responsáveis por todos os aspectos do trabalho, garantindo sua precisão e integridade. 


\section{Referências}

1. World Health Organization. Diet, nutrition and the prevention of chronic diseases. Report of a joint WHO/FAO expert consultation [Internet]. Geneva: World Health Organization; 2003 [cited 2012 Sep 15]. Available from: http://www.who.int/ dietphysicalactivity/publications/trs916/en/

2. Ministério da Saúde (BR). Política Nacional de Alimentação e Nutrição [Internet]. 2012 [citado 2011 out 12]. Disponível em: http://dab.saude.gov.br/ portaldab/pnan.php

3. Costa MS, Silva MJ. Qualidade de vida e trabalho: o que pensam os enfermeiros da rede básica de saúde. Rev Enferm. 2007 abr-jun;15(2):236-41.

4. MacFall M, Nonneman R, Rogers LQ, Mukerji V. Health care student attitudes toward the prevention of cardiovascular disease. Nurs Educ Perspect. 2009 Sep-0ct;30(5):285-9.

5. Prefeitura Municipal de Pelotas (Rio Grande do Sul). Secretaria Municipal de Saúde. Plano Municipal de Saúde 2007-2009 [Internet]. Pelotas: Secretaria Municipal de Saúde de Pelotas; 2007 [citado 2012 set 8]. Disponível em: http://www.pelotas.rs.gov.br/politica_social/saude/ arquivos/plano_municipal_saude.pdf

6. World Health Organization. Physical status: the use and interpretation of anthropometry. Report of a WHO expert committee [Internet]. Geneva: World Health Organization; 1995 [cited 2012 sep 18]. (Technical Report Series, 854) Available from: http://whqlibdoc. who.int/trs/WHO_TRS_854.pdf?ua=1

7. Ministério da Saúde (BR). Guia alimentar da alimentação para a população brasileira: promovendo a alimentação saudável [Internet] Brasília: Ministério da Saúde; 2005 [citado 2012 jul 25]. Disponível em: http://dtr2001.saude.gov.br/editora/produtos/livros/ pdf/05_1109_M.pdf

8. Sousa R, Sobral D, Paz S, Martins M. Prevalência de sobrepeso e obesidade entre funcionários plantonistas de unidades de saúde de Teresina, Piauí. Rev Nutr. 2007 set-out;20(5):473-82.

9. Sobrinho CLN, Carvalho FM, Bonfim TAS, Cirino CAS, Ferreira IS. Condições de trabalho e saúde dos médicos em Salvador, Brasil. Rev Assoc Med Bras. 2006;52(2):97-102.

10. Frank E, Segura C. Health practices of Canadian physicians. Can Fam Physician. 2009 Aug;55(8):810-11.
11. Sociedade Brasileira de Cardiologia, Sociedade Brasileira de Hipertensão, Sociedade Brasileira de Nefrologia. $5^{\circ}$ Diretrizes Brasileiras de Hipertensão Arterial [citado 2014 abr 23]. 2006 fev 13; São Paulo: Sociedade Brasileira de Cardiologia; 2006. Disponível em: http://publicacoes.cardiol.br/ consenso/2006/VDiretriz-HA.pdf

12. Hallal PC, Bauman AE, Heath GW, Kohld HW, Lee IM, Pratt M. Physical activity: more of the same is not enough. Lancet. 2012 Jul;20(3):190-1.

13. Vargas LM, Pilatti LA, Gutierrez GL. Inatividade Física e fatores associados: um estudo com trabalhadores do setor metalomecânico do município de Ponta Grossa/ PR. Rev Bras Ativ Fis Saude. 2013 jan;18(1):32-42.

14. Teixeira GSP, Rabello GM, Costa FS, Vieira FF, Rezende DF. Perfil da saúde dos médicos e do exercício profissional na cidade de Barbacena/MG. Rev Med. 2008 abr-jun;18(2):100-7.

15. Díaz-Realpe JE, Muñoz-Martínez J, Sierra-Torres CH. Factores de riesgo para enfermedad cardiovascular en trabajadores de una institución prestadora de servicios de salud, Colombia. Rev Salud Publica. 2006 ene-mar;9(1):64-75.

16. Bazargan M, Makar M, Bazargan-Hejazi S, Ani C, Wolf KE. Preventive, lifestyle and personal health behaviors among physicians. Acad Psychiatry. 2009 Jul-Aug;33(4):289-95.

17. Jansen AK, Lopes ACS, Lopes Filho JD. Fatores associados ao excesso de peso em funcionários de um Hospital Universitário. Rev Baiana Saude Publica. 2010 out-dez;34(4):951-66.

18. Ministério da Saúde (BR). Pratique saúde contra a hipertensão arterial [Internet]. 2012 [citado 2012 ago 30]. Disponível em: http://dtr2001.saude.gov.br/ pratique_saude/hipertensao.htm

19. Oshiro ML, Ferreira JS, Oshiro E. Hipertensão arterial em trabalhadores da estratégia saúde da família. Rev Bras Cien Saude. 2013 abr-jun;11(36):20-8.

20. Santos C, Portella E, Avila S, Soares E. Fatores dietéticos na prevenção e tratamento de comorbidades associadas à síndrome metabólica. Rev Nutr. 2006 mai-jun;19(3):389-401.

21. Zandonai AP, Sonobe HM, Sawada NO. Factores de riesgo alimentario para câncer colorrectal relacionado al consumo de carnes. Rev Esc Enferm. 2012;46(1):234-9. 
22. Marcondelli P, Costa THM, Schmitz BAA. Nível de atividade física e hábitos alimentares de universitários do $3^{\circ}$ ao $5^{\circ}$ semestres da área da saúde. Rev Nutr. 2008 jan-fev;21(1):39-47.

23. Pioltine MB, Paternez ACAC, Morimoto JM. Perfil dietético e risco cardiovascular em adolescentes de uma escola privada de SP. Rev Cien Saude. 2013 setdez;11(36):149-56.

24. Oliveira M, Sichieri R. Fracionamento das refeições e colesterol sérico em mulheres com dieta adicionada de frutas ou fibras. Rev Nutr. 2004 outdez;17(4):449-59.

25. Sebold LF, Radunz V, Carraro TE. Percepções sobre cuidar de si, promoção da saúde e sobrepeso entre acadêmicos de enfermagem. Esc Anna Nery. 2011 julset;15(3):536-41.
26. Ministério da Saúde (BR). Saúde feminina [Internet]. 2009 [citado 2013 mar 4]. Disponível em: http:// portal.saude.gov.br/portal/arquivos/pdf/23_07_ saude_feminina.pdf

27. Ministério da Saúde (BR). Secretaria de Políticas de Saúde. Departamento de Atenção Básica. Saúde do trabalhador [Internet]. Brasília: Ministério da Saúde; 2002 [citado 2014 abr 25]. Disponível em: http:// bvsms.saude.gov.br/bvs/publicacoes/cd03_12.pdf

\section{Recebido em 17/03/2014}

Aprovado em 06/08/2014 